\title{
More on Divisibility Criteria for Selected Primes
}

\author{
Adam Naumowicz \\ Institute of Informatics \\ University of Białystok \\ Sosnowa 64, 15-887 Białystok \\ Poland
}

\author{
Radosław Piliszek \\ Institute of Informatics \\ University of Białystok \\ Sosnowa 64, 15-887 Białystok \\ Poland
}

Summary. This paper is a continuation of [19], where the divisibility criteria for initial prime numbers based on their representation in the decimal system were formalized. In the current paper we consider all primes up to 101 to demonstrate the method presented in [7].

MSC: 11A63 03B35

Keywords: divisibility; divisibility rules; decimal digits

MML identifier: NUMERAL2, version: 8.1.02 5.17.1179

The notation and terminology used in this paper have been introduced in the following articles: [21, [25], [18, [1], [14, [12], [8], [9], [23], [17, 22], [2], [16], [19], [3], 4], [5], 6], [10], [15], [13], [26], [27], 24], and [11].

\section{Preliminaries on Finite Sequences}

In this paper $n, k, b$ denote natural numbers and $i$ denotes an integer.

Let us consider a non empty finite 0 -sequence $f$. Now we state the propositions:

(1) $f\lceil 1=\langle f(0)\rangle$.

(2) $f=\langle f(0)\rangle^{\frown} f_{l 1}$.

Now we state the proposition:

(3) Let us consider a finite 0 -sequence $f$. Then $\operatorname{mid}(f, 2$, len $f)=f_{\lfloor 1}$.

Let us consider finite natural-membered sets $X, Y$. Now we state the propositions: 
(4) If $X$ misses $Y$, then $\operatorname{dom}\left(\operatorname{Sgm}_{0} X \frown \operatorname{Sgm}_{0} Y\right)=\operatorname{dom} \operatorname{Sgm}_{0}(X \cup Y)$.

(5) $\operatorname{rng}\left(\operatorname{Sgm}_{0} X \frown \operatorname{Sgm}_{0} Y\right)=\operatorname{rng} \operatorname{Sgm}_{0}(X \cup Y)$.

Now we state the proposition:

(6) Let us consider a finite 0 -sequence $F$ and a set $X$.

Then dom the $X$-subsequence of $F=\operatorname{dom}_{\operatorname{Sgm}_{0}}(X \cap \operatorname{dom} F)$.

One can check that the functor $\mathbb{N}_{\text {even }}$ is defined by the term

(Def. 1) $\{n$, where $n$ is a natural number : $n$ is even $\}$.

Note that the functor $\mathbb{N}_{\text {odd }}$ is defined by the term

(Def. 2) $\{n$, where $n$ is a natural number : $n$ is odd $\}$.

Now we state the propositions:

(7) $\quad \mathbb{N}_{\text {even }}$ misses $\mathbb{N}_{\text {odd }}$. ProOF: $\mathbb{N}_{\text {even }} \cap \mathbb{N}_{\text {odd }} \subseteq \emptyset$

(8) $\mathbb{N}_{\text {even }} \cup \mathbb{N}_{\text {odd }}=\mathbb{N}$.

Let $F$ be a transfinite sequence and $P$ be a permutation of $\operatorname{dom} F$. One can verify that $F \cdot P$ is transfinite sequence-like.

Now we state the propositions:

(9) Let us consider a finite 0 -sequence $F$ and sets $X, Y$. Suppose $X$ misses $Y$. Then there exists a permutation $P$ of dom the $X \cup Y$-subsequence of $F$ such that (the $X \cup Y$-subsequence of $F) \cdot P=($ the $X$-subsequence of $F)^{\frown}$ (the $Y$-subsequence of $F$ ). The theorem is a consequence of (5), (4), and $(6)$.

(10) Let us consider a complex-valued finite 0 -sequence $\mathcal{F}$ and sets $B_{1}, B_{2}$. Suppose $B_{1}$ misses $B_{2}$. Then $\sum$ the $B_{1} \cup B_{2}$-subsequence of $\mathcal{F}=$ $\sum$ the $B_{1}$-subsequence of $\mathcal{F}+\sum$ the $B_{2}$-subsequence of $\mathcal{F}$. The theorem is a consequence of (9).

(11) Let us consider a finite 0 -sequence $F$. Then $F=$ the $\mathbb{N}$-subsequence of $F$.

Let us consider natural numbers $N, i$. Now we state the propositions:

(12) If $i \in \operatorname{dom}_{\operatorname{Sgm}}\left(N \cap \mathbb{N}_{\text {even }}\right)$, then $\left(\operatorname{Sgm}_{0}\left(N \cap \mathbb{N}_{\text {even }}\right)\right)(i)=2 \cdot i$.

(13) If $i \in \operatorname{dom} \operatorname{Sgm}_{0}\left(N \cap \mathbb{N}_{\text {odd }}\right)$, then $\left(\operatorname{Sgm}_{0}\left(N \cap \mathbb{N}_{\text {odd }}\right)\right)(i)=2 \cdot i+1$.

\section{Lemmas on Some Divisibility Properties}

Now we state the propositions:

(14) Let us consider integers $i, j$. Then $(i \bmod j) \bmod j=i \bmod j$.

(15) Let us consider integers $i, j, k, l$. Suppose $i \bmod l=j \bmod l$. Then $(k+i) \bmod l=(k+j) \bmod l$.

(16) Let us consider a finite 0 -sequence $d$ of $\mathbb{Z}$ and an integer $n$. Suppose a natural number $i$. If $i \in \operatorname{dom} d$, then $n \mid d(i)$. Then $n \mid \sum d$. 
(17) Let us consider finite 0 -sequences $d$, e of $\mathbb{Z}$ and an integer $n$. Suppose

(i) $\operatorname{dom} d=\operatorname{dom} e$, and

(ii) for every natural number $i$ such that $i \in \operatorname{dom} d$ holds $e(i)=d(i) \bmod$ $n$.

Then $\sum d \bmod n=\sum e \bmod n$. The theorem is a consequence of (14). Proof: Define $\mathcal{P}$ [finite 0 -sequence of $\mathbb{Z}$ ] $\equiv$ for every finite 0 -sequence $e$ of $\mathbb{Z}$ such that $\operatorname{dom} \$_{1}=\operatorname{dom} e$ and for every natural number $i$ such that $i \in \operatorname{dom} \$_{1}$ holds $e(i)=\$_{1}(i) \bmod n$ holds $\sum \$_{1} \bmod n=\sum e \bmod n$. For every finite 0 -sequence $p$ of $\mathbb{Z}$ and for every element $l$ of $\mathbb{Z}$ such that $\mathcal{P}[p]$ holds $\mathcal{P}\left[p^{\frown}\langle l\rangle\right]$ by [2, (44), (13)], [25, (33)]. $\mathcal{P}\left[\langle\rangle_{\mathbb{Z}}\right]$ by [25, (15)]. For every finite 0 -sequence $p$ of $\mathbb{Z}, \mathcal{P}[p]$ from [18, Sch. 2].

(18) Let us consider finite 0 -sequences $f, g$ of $\mathbb{N}$ and an integer $i$. Suppose

(i) $\operatorname{dom} f=\operatorname{dom} g$, and

(ii) for every element $n$ such that $n \in \operatorname{dom} f$ holds $f(n)=i \cdot g(n)$.

Then $\sum f=i \cdot \sum g$.

(19) If $b>1$, then $n=b \cdot \operatorname{value}(\operatorname{mid}(\operatorname{digits}(n, b), 2$, len $\operatorname{digits}(n, b)), b)+$ $(\operatorname{digits}(n, b))(0)$. The theorem is a consequence of (2), (18), and (3).

Let us consider natural numbers $n, k$. Now we state the propositions:

(20) If $k=10^{2 \cdot n}-1$, then $11 \mid k$.

(21) If $k=10^{2 \cdot n+1}+1$, then $11 \mid k$.

Now we state the propositions:

(22) 7 and 10 are relatively prime.

(23) 29 is prime.

(24) 31 is prime.

(25) 41 is prime.

(26) 47 is prime.

(27) 53 is prime.

(28) 59 is prime.

(29) 61 is prime.

(30) 67 is prime.

(31) 71 is prime.

(32) 73 is prime.

(33) 79 is prime.

(34) 89 is prime.

(35) 97 is prime.

(36) 101 is prime. 


\section{Divisibility Criteria for Primes up to 101}

Let us consider a prime natural number $p$ and natural numbers $n, f, b$. Now we state the propositions:

(37) Suppose there exists a natural number $k$ such that $b \cdot f+1=p \cdot k$ and $b>1$ and $p$ and $b$ are relatively prime. Then $p \mid n$ if and only if $p \mid \operatorname{value}(\operatorname{mid}(\operatorname{digits}(n, b), 2$, len $\operatorname{digits}(n, b)), b)-f \cdot(\operatorname{digits}(n, b))(0)$.

(38) Suppose there exists a natural number $k$ such that $b \cdot f-1=p \cdot k$ and $b>1$ and $p$ and $b$ are relatively prime. Then $p \mid n$ if and only if $p \mid \operatorname{value}(\operatorname{mid}(\operatorname{digits}(n, b), 2$, len $\operatorname{digits}(n, b)), b)+f \cdot(\operatorname{digits}(n, b))(0)$.

Now we state the propositions:

(39) Divisibility RUle-Divisibility By 7:

$7 \mid n$ if and only if $7 \mid \operatorname{value}(\operatorname{mid}(\operatorname{digits}(n, 10), 2, \operatorname{len} \operatorname{digits}(n, 10)), 10)-2$. $(\operatorname{digits}(n, 10))(0)$. The theorem is a consequence of $(37)$ and $(22)$.

(40) $7 \mid n$ if and only if $7 \mid \operatorname{value}\left((\operatorname{digits}(n, 10))_{\lfloor 1}, 10\right)-2 \cdot(\operatorname{digits}(n, 10))(0)$. The theorem is a consequence of (3) and (39).

(41) $11 \mid n$ if and only if $11 \mid \operatorname{value}(\operatorname{mid}(\operatorname{digits}(n, 10), 2$, len $\operatorname{digits}(n, 10)), 10)-$ $(\operatorname{digits}(n, 10))(0)$. The theorem is a consequence of $(37)$.

(42) $11 \mid n$ if and only if $11 \mid \operatorname{value}\left((\operatorname{digits}(n, 10))_{\lfloor 1}, 10\right)-(\operatorname{digits}(n, 10))(0)$. The theorem is a consequence of (3) and (41).

Now we state the proposition:

(43) Divisibility RUle-Divisibility By 11:

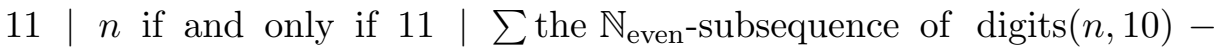
$\sum$ the $\mathbb{N}_{\text {odd-subsequence of }} \operatorname{digits}(n, 10)$. The theorem is a consequence of (10), (7), (8), (11), (6), (12), (13), (20), (16), (21), and (14). Proof: Set $d=\operatorname{digits}(n, 10)$. Consider $p$ being a finite 0 -sequence of $\mathbb{N}$ such that $\operatorname{dom} p=\operatorname{dom} d$ and for every natural number $i$ such that $i \in \operatorname{dom} p$ holds $p(i)=d(i) \cdot 10^{i}$ and value $(d, 10)=\sum p$. Set $p_{3}=$ the $\mathbb{N}_{\text {even-subsequence }}$ of $p$. Set $p_{2}=$ the $\mathbb{N}_{\text {odd }}$-subsequence of $p$. Set $d_{2}=$ the $\mathbb{N}_{\text {even-subsequence }}$ of $d$. Set $d_{3}=$ the $\mathbb{N}_{\text {odd }}$-subsequence of $d$. For every natural number $i$ such that $i \in \operatorname{dom} d_{2}$ holds $d_{2}(i)=d(2 \cdot i)$ by [8, (11), (12)]. For every natural number $i$ such that $i \in \operatorname{dom} p_{3}$ holds $p_{3}(i)=d_{2}(i) \cdot 10^{2 \cdot i}$ by [8. (11), (12)]. For every natural number $i$ such that $i \in \operatorname{dom} d_{3}$ holds $d_{3}(i)=d(2 \cdot i+1)$ by [8, (11), (12)]. For every natural number $i$ such that $i \in \operatorname{dom} p_{2}$ holds $p_{2}(i)=d_{3}(i) \cdot 10^{2 \cdot i+1}$ by [8, (11), (12)]. Define $\mathcal{E}$ [set, set] $\equiv$ $\$_{2}=p_{3}\left(\$_{1}\right)-d_{2}\left(\$_{1}\right)$. For every natural number $k$ such that $k \in \mathbb{Z}_{\operatorname{dom} p_{3}}$ there exists an element $x$ of $\mathbb{Z}$ such that $\mathcal{E}[k, x]$. Consider $p_{1}$ being a finite 0 -sequence of $\mathbb{Z}$ such that $\operatorname{dom} p_{1}=\mathbb{Z}_{\operatorname{dom} p_{3}}$ and for every natural number $k$ such that $k \in \mathbb{Z}_{\operatorname{dom} p_{3}}$ holds $\mathcal{E}\left[k, p_{1}(k)\right]$ from [20, Sch. 5]. For every natural number $i$ such that $i \in \operatorname{dom} p_{3}$ holds $p_{3}(i)=+_{\mathbb{Z}}\left(p_{1}(i), d_{2}(i)\right)$. Define $\mathcal{O}$ [set, set $] \equiv \$_{2}=p_{2}\left(\$_{1}\right)+d_{3}\left(\$_{1}\right)$. Consider $p_{4}$ being a finite 0 -sequence of 
$\mathbb{N}$ such that $\operatorname{dom} p_{4}=\mathbb{Z}_{\operatorname{dom} p_{2}}$ and for every natural number $k$ such that $k \in \mathbb{Z}_{\text {dom } p_{2}}$ holds $\mathcal{O}\left[k, p_{4}(k)\right]$ from [20, Sch. 5]. Set $m=(-1) \cdot d_{3}$. For every natural number $i$ such that $i \in \operatorname{dom} p_{2}$ holds $p_{2}(i)=+_{\mathbb{Z}}\left(p_{4}(i), m(i)\right)$. If $11 \mid n$, then $11 \mid \sum d_{2}-\sum d_{3}$ by [19, (5)], [23, (62)]. If $11 \mid \sum d_{2}-\sum d_{3}$, then $11 \mid n$ by [23, (62)], [19, (5)].

Now we state the propositions:

(44) Divisibility RUle-Divisibility By 13:

$13 \mid n$ if and only if $13 \mid \operatorname{value}(\operatorname{mid}(\operatorname{digits}(n, 10), 2, \operatorname{len} \operatorname{digits}(n, 10)), 10)+$ $4 \cdot(\operatorname{digits}(n, 10))(0)$. The theorem is a consequence of $(38)$.

(45) $13 \mid n$ if and only if $13 \mid \operatorname{value}\left((\operatorname{digits}(n, 10))_{\downarrow 1}, 10\right)+4 \cdot(\operatorname{digits}(n, 10))(0)$. The theorem is a consequence of (3) and (44).

(46) $\quad 17 \mid n$ if and only if $17 \mid \operatorname{value}(\operatorname{mid}(\operatorname{digits}(n, 10), 2$, len $\operatorname{digits}(n, 10)), 10)-$ $5 \cdot(\operatorname{digits}(n, 10))(0)$. The theorem is a consequence of $(37)$.

(47) $17 \mid n$ if and only if $17 \mid \operatorname{value}\left((\operatorname{digits}(n, 10))_{\lfloor 1}, 10\right)-5 \cdot(\operatorname{digits}(n, 10))(0)$. The theorem is a consequence of (3) and (46).

(48) $19 \mid n$ if and only if $19 \mid \operatorname{value}(\operatorname{mid}(\operatorname{digits}(n, 10), 2$, len $\operatorname{digits}(n, 10)), 10)+$ $2 \cdot(\operatorname{digits}(n, 10))(0)$. The theorem is a consequence of $(38)$.

(49) $19 \mid n$ if and only if $19 \mid \operatorname{value}\left((\operatorname{digits}(n, 10))_{\downarrow 1}, 10\right)+2 \cdot(\operatorname{digits}(n, 10))(0)$. The theorem is a consequence of (3) and (48).

(50) $23 \mid n$ if and only if $23 \mid \operatorname{value}(\operatorname{mid}(\operatorname{digits}(n, 10), 2$, len $\operatorname{digits}(n, 10)), 10)+$ $7 \cdot(\operatorname{digits}(n, 10))(0)$. The theorem is a consequence of $(38)$.

(51) $23 \mid n$ if and only if $23 \mid \operatorname{value}\left((\operatorname{digits}(n, 10))_{\downarrow 1}, 10\right)+7 \cdot(\operatorname{digits}(n, 10))(0)$. The theorem is a consequence of (3) and (50).

(52) $29 \mid n$ if and only if $29 \mid \operatorname{value}(\operatorname{mid}(\operatorname{digits}(n, 10), 2$, len $\operatorname{digits}(n, 10)), 10)+$ $3 \cdot(\operatorname{digits}(n, 10))(0)$. The theorem is a consequence of $(23)$ and (38).

(53) $29 \mid n$ if and only if $29 \mid \operatorname{value}\left((\operatorname{digits}(n, 10))_{\downarrow 1}, 10\right)+3 \cdot(\operatorname{digits}(n, 10))(0)$. The theorem is a consequence of (3) and (52).

(54) $31 \mid n$ if and only if $31 \mid \operatorname{value}(\operatorname{mid}(\operatorname{digits}(n, 10), 2$, len $\operatorname{digits}(n, 10)), 10)-$ $3 \cdot(\operatorname{digits}(n, 10))(0)$. The theorem is a consequence of $(24)$ and $(37)$.

(55) $31 \mid n$ if and only if $31 \mid \operatorname{value}\left((\operatorname{digits}(n, 10))_{\downarrow 1}, 10\right)-3 \cdot(\operatorname{digits}(n, 10))(0)$. The theorem is a consequence of (3) and (54).

(56) $37 \mid n$ if and only if $37 \mid \operatorname{value}(\operatorname{mid}(\operatorname{digits}(n, 10), 2$, len $\operatorname{digits}(n, 10)), 10)-$ $11 \cdot(\operatorname{digits}(n, 10))(0)$. The theorem is a consequence of $(37)$.

(57) $37 \mid n$ if and only if $37 \mid \operatorname{value}\left((\operatorname{digits}(n, 10))_{\lfloor 1}, 10\right)-11 \cdot(\operatorname{digits}(n, 10))(0)$. The theorem is a consequence of (3) and (56).

(58) $\quad 41 \mid n$ if and only if $41 \mid \operatorname{value}(\operatorname{mid}(\operatorname{digits}(n, 10), 2$, len $\operatorname{digits}(n, 10)), 10)-$ $4 \cdot(\operatorname{digits}(n, 10))(0)$. The theorem is a consequence of $(25)$ and $(37)$.

(59) $41 \mid n$ if and only if $41 \mid \operatorname{value}\left((\operatorname{digits}(n, 10))_{\downarrow 1}, 10\right)-4 \cdot(\operatorname{digits}(n, 10))(0)$. The theorem is a consequence of (3) and (58). 
(60) $43 \mid n$ if and only if $43 \mid \operatorname{value}(\operatorname{mid}(\operatorname{digits}(n, 10), 2, \operatorname{len} \operatorname{digits}(n, 10)), 10)+$ $13 \cdot(\operatorname{digits}(n, 10))(0)$. The theorem is a consequence of $(38)$.

(61) $43 \mid n$ if and only if $43 \mid \operatorname{value}\left((\operatorname{digits}(n, 10))_{\downarrow 1}, 10\right)+13 \cdot(\operatorname{digits}(n, 10))(0)$. The theorem is a consequence of (3) and (60).

(62) $\quad 47 \mid n$ if and only if $47 \mid \operatorname{value}(\operatorname{mid}(\operatorname{digits}(n, 10), 2$, len $\operatorname{digits}(n, 10)), 10)-$ $14 \cdot(\operatorname{digits}(n, 10))(0)$. The theorem is a consequence of $(26)$ and $(37)$.

(63) $47 \mid n$ if and only if $47 \mid \operatorname{value}\left((\operatorname{digits}(n, 10))_{\mid 1}, 10\right)-14 \cdot(\operatorname{digits}(n, 10))(0)$. The theorem is a consequence of (3) and (62).

(64) $53 \mid n$ if and only if $53 \mid \operatorname{value}(\operatorname{mid}(\operatorname{digits}(n, 10), 2$, len $\operatorname{digits}(n, 10)), 10)+$ $16 \cdot(\operatorname{digits}(n, 10))(0)$. The theorem is a consequence of $(27)$ and $(38)$.

(65) $53 \mid n$ if and only if $53 \mid \operatorname{value}\left((\operatorname{digits}(n, 10))_{\lfloor 1}, 10\right)+16 \cdot(\operatorname{digits}(n, 10))(0)$. The theorem is a consequence of (3) and (64).

(66) $59 \mid n$ if and only if $59 \mid \operatorname{value}(\operatorname{mid}(\operatorname{digits}(n, 10), 2, \operatorname{len} \operatorname{digits}(n, 10)), 10)+$ $6 \cdot(\operatorname{digits}(n, 10))(0)$. The theorem is a consequence of $(28)$ and $(38)$.

(67) $59 \mid n$ if and only if $59 \mid \operatorname{value}\left((\operatorname{digits}(n, 10))_{\lfloor 1}, 10\right)+6 \cdot(\operatorname{digits}(n, 10))(0)$. The theorem is a consequence of (3) and (66).

(68) $\quad 61 \mid n$ if and only if $61 \mid \operatorname{value}(\operatorname{mid}(\operatorname{digits}(n, 10), 2$, len $\operatorname{digits}(n, 10)), 10)-$ $6 \cdot(\operatorname{digits}(n, 10))(0)$. The theorem is a consequence of $(29)$ and $(37)$.

(69) $61 \mid n$ if and only if $61 \mid \operatorname{value}\left((\operatorname{digits}(n, 10))_{\lfloor 1}, 10\right)-6 \cdot(\operatorname{digits}(n, 10))(0)$. The theorem is a consequence of (3) and (68).

(70) $\quad 67 \mid n$ if and only if $67 \mid \operatorname{value}(\operatorname{mid}(\operatorname{digits}(n, 10), 2$, len $\operatorname{digits}(n, 10)), 10)-$ $20 \cdot(\operatorname{digits}(n, 10))(0)$. The theorem is a consequence of (30) and (37).

(71) $67 \mid n$ if and only if $67 \mid \operatorname{value}\left((\operatorname{digits}(n, 10))_{\downarrow 1}, 10\right)-20 \cdot(\operatorname{digits}(n, 10))(0)$. The theorem is a consequence of $(3)$ and (70).

(72) $\quad 71 \mid n$ if and only if $71 \mid \operatorname{value}(\operatorname{mid}(\operatorname{digits}(n, 10), 2$, len $\operatorname{digits}(n, 10)), 10)-$ $7 \cdot(\operatorname{digits}(n, 10))(0)$. The theorem is a consequence of (31) and (37).

(73) $71 \mid n$ if and only if $71 \mid \operatorname{value}\left((\operatorname{digits}(n, 10))_{\lfloor 1}, 10\right)-7 \cdot(\operatorname{digits}(n, 10))(0)$. The theorem is a consequence of (3) and (72).

(74) $73 \mid n$ if and only if $73 \mid \operatorname{value}(\operatorname{mid}(\operatorname{digits}(n, 10), 2$, len $\operatorname{digits}(n, 10)), 10)+$ $22 \cdot(\operatorname{digits}(n, 10))(0)$. The theorem is a consequence of $(32)$ and (38).

(75) $73 \mid n$ if and only if $73 \mid \operatorname{value}\left((\operatorname{digits}(n, 10))_{\downarrow 1}, 10\right)+22 \cdot(\operatorname{digits}(n, 10))(0)$. The theorem is a consequence of (3) and (74).

(76) $79 \mid n$ if and only if $79 \mid \operatorname{value}(\operatorname{mid}(\operatorname{digits}(n, 10), 2$, len $\operatorname{digits}(n, 10)), 10)+$ $8 \cdot(\operatorname{digits}(n, 10))(0)$. The theorem is a consequence of (33) and (38).

(77) $79 \mid n$ if and only if $79 \mid \operatorname{value}\left((\operatorname{digits}(n, 10))_{\lfloor 1}, 10\right)+8 \cdot(\operatorname{digits}(n, 10))(0)$. The theorem is a consequence of (3) and (76).

(78) $83 \mid n$ if and only if $83 \mid \operatorname{value}(\operatorname{mid}(\operatorname{digits}(n, 10), 2$, len $\operatorname{digits}(n, 10)), 10)+$ $25 \cdot(\operatorname{digits}(n, 10))(0)$. The theorem is a consequence of $(38)$. 
(79) $83 \mid n$ if and only if $83 \mid \operatorname{value}\left((\operatorname{digits}(n, 10))_{\downarrow 1}, 10\right)+25 \cdot(\operatorname{digits}(n, 10))(0)$. The theorem is a consequence of (3) and (78).

(80) $89 \mid n$ if and only if $89 \mid \operatorname{value}(\operatorname{mid}(\operatorname{digits}(n, 10), 2, \operatorname{len} \operatorname{digits}(n, 10)), 10)+$ $9 \cdot(\operatorname{digits}(n, 10))(0)$. The theorem is a consequence of $(34)$ and $(38)$.

(81) $89 \mid n$ if and only if $89 \mid \operatorname{value}\left((\operatorname{digits}(n, 10))_{\lfloor 1}, 10\right)+9 \cdot(\operatorname{digits}(n, 10))(0)$. The theorem is a consequence of (3) and (80).

(82) $97 \mid n$ if and only if $97 \mid \operatorname{value}(\operatorname{mid}(\operatorname{digits}(n, 10), 2$, len $\operatorname{digits}(n, 10)), 10)-$ $29 \cdot(\operatorname{digits}(n, 10))(0)$. The theorem is a consequence of $(35)$ and $(37)$.

(83) $97 \mid n$ if and only if $97 \mid \operatorname{value}\left((\operatorname{digits}(n, 10))_{\lfloor 1}, 10\right)-29 \cdot(\operatorname{digits}(n, 10))(0)$. The theorem is a consequence of (3) and (82).

(84) $101 \mid n$ if and only if $101 \mid \operatorname{value}(\operatorname{mid}(\operatorname{digits}(n, 10), 2, \operatorname{len} \operatorname{digits}(n, 10)), 10)-$ $10 \cdot(\operatorname{digits}(n, 10))(0)$. The theorem is a consequence of $(36)$ and $(37)$.

(85) $101 \mid n$ if and only if $101 \mid \operatorname{value}\left((\operatorname{digits}(n, 10))_{\lfloor 1}, 10\right)-10 \cdot(\operatorname{digits}(n, 10))(0)$. The theorem is a consequence of (3) and (84).

\section{REFERENCES}

[1] Grzegorz Bancerek. Cardinal numbers. Formalized Mathematics, 1(2):377-382, 1990.

[2] Grzegorz Bancerek. The fundamental properties of natural numbers. Formalized Mathematics, 1(1):41-46, 1990.

[3] Grzegorz Bancerek. The ordinal numbers Formalized Mathematics, 1(1):91-96, 1990.

[4] Grzegorz Bancerek. Sequences of ordinal numbers Formalized Mathematics, 1(2):281290, 1990.

[5] Grzegorz Bancerek. Increasing and continuous ordinal sequences, Formalized Mathematics, 1(4):711-714, 1990.

[6] Grzegorz Bancerek. Veblen hierarchy. Formalized Mathematics, 19(2):83-92, 2011. doi $10.2478 / v 10037-011-0014-5$

[7] C.C. Briggs. Simple divisibility rules for the 1st 1000 prime numbers arXiv preprint arXiv:math/0001012, 2000.

[8] Czesław Byliński. Functions and their basic properties Formalized Mathematics, 1(1): 55-65, 1990.

[9] Czesław Byliński. Functions from a set to a set. Formalized Mathematics, 1(1):153-164, 1990.

[10] Czesław Byliński. Partial functions. Formalized Mathematics, 1(2):357-367, 1990.

[11] Czesław Byliński. Some basic properties of sets. Formalized Mathematics, 1(1):47-53, 1990.

[12] Agata Darmochwał. Finite sets Formalized Mathematics, 1(1):165-167, 1990.

[13] Krzysztof Hryniewiecki. Recursive definitions Formalized Mathematics, 1(2):321-328, 1990.

[14] Magdalena Jastrzȩbska and Adam Grabowski. Some properties of Fibonacci numbers Formalized Mathematics, 12(3):307-313, 2004.

[15] Artur Korniłowicz. On the real valued functions Formalized Mathematics, 13(1):181-187, 2005.

[16] Rafał Kwiatek. Factorial and Newton coefficients Formalized Mathematics, 1(5):887-890, 1990.

[17] Rafał Kwiatek and Grzegorz Zwara. The divisibility of integers and integer relatively primes Formalized Mathematics, 1(5):829-832, 1990.

[18] Yatsuka Nakamura and Hisashi Ito. Basic properties and concept of selected subsequence of zero based finite sequences. Formalized Mathematics, 16(3):283-288, 2008. doi $10.2478 / v 10037-008-0034-y$ 
[19] Adam Naumowicz. On the representation of natural numbers in positional numeral systems. Formalized Mathematics, 14(4):221-223, 2006. doi 10.2478/v10037-006-0025-9.

[20] Karol Pąk. Stirling numbers of the second kind. Formalized Mathematics, 13(2):337-345, 2005.

[21] Piotr Rudnicki and Andrzej Trybulec. Abian's fixed point theorem. Formalized Mathematics, 6(3):335-338, 1997.

[22] Andrzej Trybulec. On the sets inhabited by numbers. Formalized Mathematics, 11(4): 341-347, 2003.

[23] Michał J. Trybulec. Integers Formalized Mathematics, 1(3):501-505, 1990.

[24] Zinaida Trybulec. Properties of subsets. Formalized Mathematics, 1(1):67-71, 1990.

[25] Tetsuya Tsunetou, Grzegorz Bancerek, and Yatsuka Nakamura. Zero-based finite sequences Formalized Mathematics, 9(4):825-829, 2001.

[26] Edmund Woronowicz. Relations and their basic properties. Formalized Mathematics, 1 (1):73-83, 1990 .

[27] Edmund Woronowicz. Relations defined on sets. Formalized Mathematics, 1(1):181-186, 1990.

Received May 19, 2013 